\title{
The prevalence of primary headache disorders in Nepal: a nationwide population-based study
}

\author{
Kedar Manandhar ${ }^{1,2}$, Ajay Risal ${ }^{1,2}$, Timothy J Steiner ${ }^{1,3}$, Are Holen $^{1,4}$ and Mattias Linde ${ }^{1,5^{*}}$
}

\begin{abstract}
Background: Headache disorders are among the most prevalent and burdensome global public-health problems. Within countries, health policy depends upon knowledge of health within the local populations, but the South-East Asia Region (SEAR), among WHO's six world regions, is the only one for which no national headache prevalence data are available.
\end{abstract}

Methods: In a cross-sectional population-based study, adults representative of the Nepali-speaking population aged 18-65 years and living in Nepal were randomly recruited using stratified multistage cluster sampling. They were visited unannounced at home by trained interviewers who used a culturally-adapted Nepali translation of the structured Headache-Attributed Restriction, Disability, Social Handicap and Impaired Participation (HARDSHIP) questionnaire.

Results: There were 2,100 participants (1,239 females [59.0\%], 861 males [41.0\%]; mean age $36.4 \pm 12.8$ years) with 9 refusals (participation rate $99.6 \%)$. Over half $(1,100 ; 52.4 \%)$ were resident above 1,000 $\mathrm{m}$ and almost one quarter (470; $22.4 \%$ ) lived at or above 2,000 m. The 1-year prevalence of any headache was $85.4 \pm 1.5 \%$ (gender- and age-adjusted $84.9 \%$ ), of migraine $34.7 \pm 2.0 \%$ (34.1\%), of tension-type headache (TH) $41.1 \pm 2.1 \%$ (41.5\%), of headache on $\geq 15$ days/month $7.7 \pm 1.1 \%(7.4 \%)$ and of probable medication-overuse headache $(\mathrm{pMOH})$ $2.2 \pm 0.63 \%(2.1 \%)$.

There was a strong association between migraine and living at altitude $\geq 1,000 \mathrm{~m}$ ( $\mathrm{AOR}=1.6$ [95 \% Cl: 1.3-2.0]; $p<0.001$ ). There was a less strong association between $\mathrm{TTH}$ and urban dwelling ( $\mathrm{AOR}=1.3$ [95\% Cl: 1.1-1.6]; $p=0.003)$, and a possibly artefactual negative association between $\mathrm{TH}$ and living above $1,000 \mathrm{~m}(\mathrm{AOR}=0.7$ [95 \% Cl: 0.6-0.8]; $p<0.001)$.

Conclusion: Headache disorders are very common in Nepal. Migraine is unusually so, and strongly associated with living at altitude, which in very large part accounts for the high national prevalence: the age- and genderstandardised prevalence in the low-lying Terai is $27.9 \%$. Headache occurring on $\geq 15$ days/month is also common. This new evidence will inform national health policy and provide a basis for health-care needs assessment. However, research is needed to explain the association between migraine and altitude, since it may be relevant to health-care interventions.

Keywords: Migraine, Tension-type headache, Medication-overuse headache, Public health, population-based study, prevalence, Nepal, South-East Asia Region, Global campaign against headache

\footnotetext{
* Correspondence: mattias.linde@ntnu.no

'Department of Neuroscience, Norwegian University of Science and

Technology, St Olavs University Hospital, Trondheim, Norway

${ }^{5}$ Norwegian Advisory Unit on Headaches, St Olavs University Hospital,

Trondheim, Norway

Full list of author information is available at the end of the article
}

\section{至 Springer}

(c) 2015 Manandhar et al. Open Access This article is distributed under the terms of the Creative Commons Attribution 4.0 International License (http://creativecommons.org/licenses/by/4.0/), which permits unrestricted use, distribution, and reproduction in any medium, provided you give appropriate credit to the original author(s) and the source, provide a link to the Creative Commons license, and indicate if changes were made. 


\section{Background}

Headache disorders are among the most prevalent, burdensome and costly diseases in the world [1]. The primary headache disorders, mostly migraine and tension-type headache (TTH), are of importance to global public health because they lead to widespread ill health and impaired quality of life [2], and important to global economies because they also cause much loss of productivity [3]. Improper treatment of migraine or TTH can lead to medication-overuse headache $(\mathrm{MOH})$, an aggravated disorder which, by definition, occurs on $\geq 15$ days/month and is a major additional contributor to global disability. The Global Burden of Disease Study 2013 (GBD2013) found migraine to be the sixth highest cause of disability worldwide and $\mathrm{MOH}$ the $18^{\text {th }}$ in terms of years of life lost to disability (YLDs) [4]. Collectively, headache disorders rank third [5].

Headache prevalence is poorly described in many large and populous regions of the world. Nowhere is this more obvious than in the South-East Asia Region (SEAR), the only one of the World Health Organization's six world regions for which no nationwide data have yet been gathered about the prevalence of headache disorders or their impact on society [6].

Within SEAR, Nepal is one of the poorest countries [7]. Its population is approximately 30 million [8], of whom about one quarter live below the international poverty line and among the others the distribution of wealth is rather unequal [7]. Furthermore, Nepal is a country of wide diversity. More than 70 ethnic groups maintain different cultures and spoken languages [9]. Topographically the country is divided into three physiographic divisions - Terai, Hill and Mountain [10] - rising from some $60 \mathrm{~m}$ above sea level to the Himalayas, including Mount Everest, the highest peak on Earth at 8,848 m [7], to attest Nepal's extraordinary geographical variation.

Although headache has been found to be one of the most common complaints among patients in primary health-care centres in Nepal $[11,12]$, no epidemiological studies have established the prevalence of headache disorders in this country. Epidemiological data are required to inform policy and decide the efficient allocation of resources in a country such as Nepal which has a very limited health-care budget. With this purpose, the aim of our study was two-fold: 1) to estimate the prevalence of headache disorders of public-health importance: migraine, TTH and $\mathrm{MOH}$; and 2) to explore demographic and environmental factors associated with these headache disorders in Nepal. The study was conducted as a project within the Global Campaign against Headache, which is led by Lifting The Burden (LTB), a UK-registered non-governmental organisation in official relationship with the World Health Organization.

\section{Methods}

\section{Ethics}

The Nepal Health Research Council, the Institutional Review Committee of Kathmandu University School of Medical Sciences, Dhulikhel Hospital (IRC-KUSMS) and the Regional Committee for Health and Research Ethics in central Norway all approved the study protocol.

All participants were informed about the nature and purpose of the study. Written consent was obtained by signature or fingerprint in accordance with requirements of IRC-KUSMS.

\section{Study design}

This was a cross-sectional, population-based survey using structured interviews administered by trained health workers making unannounced door-to-door visits to households in May, 2013. We randomly selected one eligible adult (aged 18-65 years, Nepali-speaking and living in Nepal) from each household. To ensure adequate representation from the country as a whole, we used multistage stratified cluster sampling, including all three physiographic divisions and, within each, all five development regions (Far-Western, Midwestern, Western, Central and Eastern). The details of the sampling and data collection procedure, including the steps taken to ensure a very high participation rate, have been published elsewhere [10]. The sample size $(\mathrm{N}=2,100)$ was estimated assuming a headache-type prevalence of $\geq 10 \%$ and absolute margin of error of $1.3 \%$ with $95 \%$ confidence interval (CI).

\section{Instruments}

We used the Headache-Attributed Restriction, Disability, Social Handicap and Impaired Participation (HARDSHIP) questionnaire developed by LTB for similar studies [13]. The English version was translated into Nepalese according to LTB's translation protocol for lay documents [14] and adapted to fit the characteristics of the Nepalese culture [15].

The full questionnaire has been published previously [10]. It consisted of five parts. For all participants there were (i) personal and demographic enquiry and (ii) a headache screening question ("Have you had a headache in the last year?"). Those who answered "no" were classified as headache-free. Those who answered "yes" were asked whether their headaches were of one or more types and, if more than one, to focus only on the most bothersome type. Only those who answered positively to the screening question were also asked (iii) diagnostic and (iv) burden and health-care questions relating to their headache. To the standard HARDSHIP questions we added others relating to use of herbal therapies. Finally, there were (v) questions on certain comorbidities asked of all participants [10]. 
We used culturally-validated Nepali-translations of the Hospital Anxiety and Depression Scale (HADS) [16] and the Eysenck Personality Questionnaire Neuroticism Short Form Revised version (EPQRS-N) [17] to assess psychiatric comorbidity. We measured height, weight and waist-circumference, and calculated body-mass index (BMI). We measured blood pressure (BP) using a digital device (3BM1-3 ${ }^{\circ}$ by Microlife).

We recorded the altitude of each household using a portable altimeter (SAL $7030^{\circ}$ by Sunoh).

\section{Headache diagnosis}

Diagnoses were not made during the interviews but later by an algorithm [13]. Participants reporting headache on $\geq 15$ days/month were first separated as a distinct group because they cannot be fully diagnosed by questionnaire. Those who were also overusing acute medication were considered to have probable $\mathrm{MOH}(\mathrm{pMOH})$; the remainder were diagnosed as "other headache on $\geq 15$ days/month". Medication overuse was diagnosed in those who: a) reported the use on $\geq 15$ days/month of either a single class of analgesics, or one type of herbal medicine, as acute headache treatment; or b) reported using on $\geq 10$ days/month (i) a combination of analgesics of different classes, or (ii) more than one type of herbal medicine, or (iii) a combination of analgesics and herbal medicines. Triptans and ergots were not used.

To all others, reporting headache on $\leq 14$ days/ month, the algorithm applied modified criteria of the International Classification of Headache Disorders (ICHD-3 beta) [18] in the following order: definite migraine, definite $\mathrm{TTH}$, probable migraine and probable TTH. We found that two additional adaptations were necessary. Firstly, photophobia was reported in association with more than three-quarters $(75.8 \%)$ of all headaches and therefore offered little discriminative value diagnostically. Accordingly, we ignored it when diagnosing headache types. Secondly, according to ICHD-3 beta [18], attacks lasting $<4 \mathrm{~h}$ when untreated in adults may be compatible with a diagnosis of probable migraine when other criteria are met. Many of our participants could report attack durations only after taking acute medication, and some were very short. We decided to disallow a diagnosis of probable migraine (in favour of probable TTH) whenever headache duration was $<1 \mathrm{~h}$. We took the view that so short a duration, even with acute treatment, was very unlikely to be migraine, given that the adult participant was asked to describe a "usual" attack [19].

Cases of definite and probable migraine were combined as, likewise, were cases of definite and probable TTH in the estimations of prevalence and in association analyses. Remaining cases were considered unclassifiable.

\section{Statistics}

We estimated crude 1-year prevalence with $95 \%$ CI for all headache, migraine and $\mathrm{TTH}$, and point prevalence for all headache on $\geq 15$ days/month and $\mathrm{pMOH}$. We adjusted prevalences for gender and age according to the general population distribution (within the range 18-65 years) in Nepal [20].

We categorized age in five groups (18-25, 26-35, $36-45,46-55$ and 56-65 years). We classed habitation as rural or urban, and categorized altitude of the household as $<500 \mathrm{~m}, 500-999 \mathrm{~m}, 1,000-1,499 \mathrm{~m}$, 1,500-1,999 m, 2,000-2,499 $\mathrm{m}$ or $\geq 2,500 \mathrm{~m}$. We took household consumption per year in USD (at the time of the survey, USD $1=$ NPR 100) as an indicator of the economic wellbeing of the participant and categorized it in three groups: poorest (<USD 950/year); poor (USD 950-1,200/year); intermediate and above (>USD 1,200/year).

We used bivariate and multivariate logistic regression analyses (with odds ratios [ORs] and adjusted ORs [AORs] respectively, each with $95 \% \mathrm{CIs}$ ) to investigate associations of demographic, lifestyle, environmental and other health factors with each of migraine, TTH and pMOH. Gender, age, household consumption, habitation, altitude, systolic and diastolic BP and EPQRS-N and HADS scores were entered as covariates in the multivariate logistic regression. BP readings and EPQRS-N and HADS scores were treated as continuous variables.

Statistical analyses were carried out using the Statistical Package for Social Science software (IBM SPSS Statistics 21, Chicago, USA).

\section{Results}

The survey was completed by 2,100 participants $(1,239$ [59.0\%] female, 861 [41.0\%] male) aged 18-65 years (mean age $36.4 \pm 12.8$ years). There were only nine refusals: hence the participation rate was $99.6 \%$. Almost two-fifths $(822 ; 39.1 \%)$ were living in households with the poorest economic wellbeing; nearly two thirds $(1,328 ; 63.2 \%)$ were from rural areas; over half $(1,100$; $52.4 \%)$ were resident above $1,000 \mathrm{~m}$ and almost one quarter $(470 ; 22.4 \%)$ lived at or above $2,000 \mathrm{~m}$. The sociodemographic characteristics of the sample are compared with those of the national population (as far as they are available) from the 2011 population and housing census [20] in Table 1.

\section{Headache prevalences \\ All headache}

Of the 2,100 participants, 1,794 reported headache in the last year. The crude 1-year prevalence of all headache was $85.4 \%$ (95 \% CI: 83.9-86.9\%), higher in females $(89.2 \%)$ than in males $(80.0 \% ; p<0.001)$. The 
Table 1 Sociodemographic characteristics of the participating sample $(N=2,100)$ and national population

\begin{tabular}{lll}
\hline Variable & $\begin{array}{l}\text { Sample } \\
n(\%)\end{array}$ & $\begin{array}{l}\text { National population [20] } \\
\text { Gender }\end{array}$ \\
$\quad$ Male & $861(41.0)$ & 46.6 \\
Female & $1,239(59.0)$ & 53.4 \\
Age (years) & & \\
18-25 & $489(23.3)$ & 27.7 \\
$26-35$ & $657(31.3)$ & 26.1 \\
36-45 & $438(20.8)$ & 20.4 \\
$46-55$ & $298(14.2)$ & 14.8 \\
56-65 & $218(10.4)$ & 11.0 \\
Household consumption & & \\
(USD/year) & & \\
$<950$ & $822(39.1)$ & Data not available \\
950-1200 & $806(38.4)$ & \\
$>1,200$ & $472(22.5)$ & \\
Habitation & & \\
Rural & $1,328(63.2)$ & 72.8 \\
Urban & $772(36.8)$ & 27.2 \\
Household altitude & & \\
$<1,000 \mathrm{~m}$ & $1,000(47.6)$ & Data not available \\
$\geq 1,000 \mathrm{~m}$ & & \\
\hline
\end{tabular}

gender- and age-adjusted 1-year prevalence was $84.9 \%$. The age-adjusted female-to-male ratio was 1.1 .

\section{Migraine}

The crude 1-year prevalence of migraine was $34.7 \%$ (17.5\% definite, $17.2 \%$ probable). Prevalence was agerelated, increasing from young adulthood (18-25 years) in both genders and peaking during 26-35 years among males and 36-45 years among females (Fig. 1).

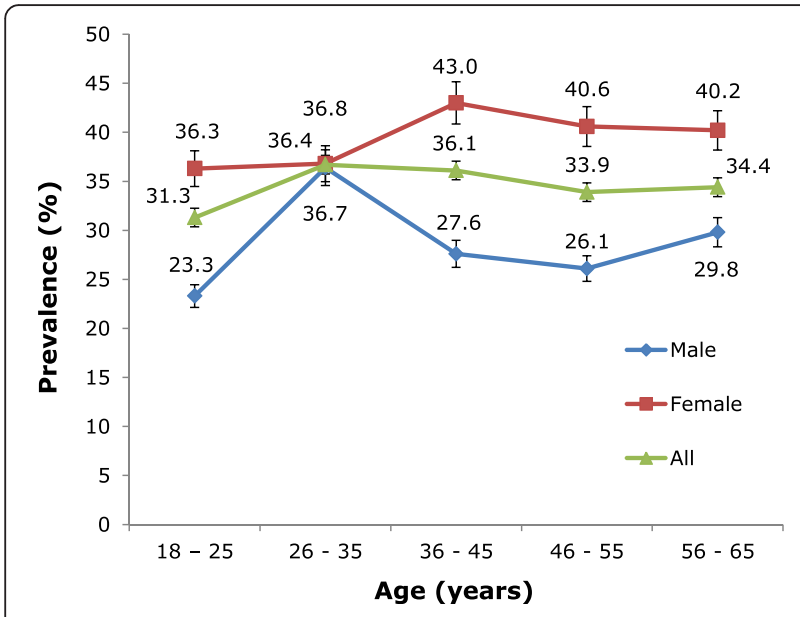

Fig. 1 1-year prevalence of migraine by age and gender
There was a clear female preponderance (overall $38.2 \%$ versus $28.9 \%$ in males) (Table 2 ), which is demonstrated in Fig. 1 in all age groups except 26-35 years and was confirmed in both bivariate $(\mathrm{OR}=1.6$ [95\% CI: 1.3-1.9]; $\mathrm{p}<0.001$ ) (Table 3) and multivariate regression analysis $\quad(\mathrm{AOR}=1.5 \quad[95 \quad \% \quad \mathrm{CI}: 1.2-1.8] ; \quad p<0.001)$ (Table 4). The gender- and age-adjusted 1-year prevalence was $34.1 \%$ and the age-adjusted female-to-male ratio was 1.3 .

\section{Tension-type headache}

The crude 1-year prevalence of TTH was $41.1 \%$ (32.5\% definite, $8.6 \%$ probable). Prevalence was lower in females $(38.7 \%)$ than in males $(44.6 \%$; OR $=0.7$ [95 \% CI: $0.6-0.9]$; $p=0.002$ ) (Tables 2,3 ). The gender- and ageadjusted prevalence was $41.5 \%$ and the age-adjusted female-to-male ratio was 0.86 . Prevalence was highest in the age range 18-25 years and decreased with age in both genders, being at its lowest at 56-65 years (OR = 0.6 [95\% CI: $0.4-0.8] ; p<0.001$ ) (Table 3). Multivariate regression analysis (Table 4) confirmed that TTH was negatively associated with female gender $(\mathrm{AOR}=0.7$ [95 \% CI: $0.6-0.9] ; \quad p=0.003$ ) and age (for age 56-65 years, $\mathrm{AOR}=0.6$ [95 \% CI: 0.4-0.9]; $p=0.008$ ).

\section{Headache on $\geq 15$ days/month and $\mathrm{pMOH}$}

The crude prevalence of all headache on $\geq 15$ days/month was $7.7 \%$ (95\% CI: 6.6-8.8\%), higher in females (9.4\%) than males $(5.1 \% ; p<0.001)$. The age-adjusted female-tomale ratio was 1.86 . The gender- and age-adjusted prevalence was $7.4 \%$.

Over one quarter $(46 / 161 ; 28.6 \%)$ of the participants reporting headache on $\geq 15$ days/month fulfilled our criteria for $\mathrm{pMOH}$. The crude prevalence of $\mathrm{pMOH}$ was therefore $2.2 \%$ (95 \% CI: 1.6-2.8). This disorder was much more common in females $(2.8 \%)$ than males (1.3\%; OR $=2.2$ [95 \% CI: $1.1-4.4] ; p=0.020 ; \mathrm{AOR}=2.6$ [95 \% CI: $1.2-5.3$ ]; $p=0.010)$. The age-adjusted femaleto-male ratio was 2.42 (Tables 2, 3, 4). Prevalence increased with age and was highest in the age range 56-65 years $(\mathrm{OR}=3.7$ [95 \% CI: 1.2-11.4]; $p=0.023$ ) (Table 3). The gender- and age-adjusted prevalence was $2.1 \%$.

The most commonly overused acute treatment was paracetamol in monotherapy (26 cases [56.5\%]). In 6 cases $(16.1 \%)$, there was overuse of herbal medicines, usually navaratna sancho and zandubalm, which are customarily inhaled or administered via the nasal mucosa for treatment of headache in Nepal.

\section{Associations with household consumption, habitation, and household altitude}

None of the headache disorders was associated with household consumption (Table 4). TTH was weakly associated with urban dwelling $(\mathrm{OR}=1.4$ [95 \% CI: 
Table 2 Observed 1-year prevalence (\% [95 \% CI]) of all headache and headache types by gender, age, household consumption, habitation and altitude

\begin{tabular}{|c|c|c|c|c|c|}
\hline & All headache & Migraine & Tension-type headache & All headache on $\geq 15$ days/month & Probable medication-overuse headache \\
\hline \multicolumn{6}{|l|}{ Gender } \\
\hline Male & $80.0[77.2-82.6]$ & $28.9[25.9-31.9]$ & $44.6[41.3-47.9]$ & $5.1[3.6-6.6]$ & $1.3[0.5-2.1]$ \\
\hline Female & $89.2[87.3-90.8]$ & $38.2[35.5-40.9]$ & $38.7[36.0-41.4]$ & $9.4[7.8-11.0]$ & $2.8[1.9-3.7]$ \\
\hline \multicolumn{6}{|l|}{ Age (years) } \\
\hline $18-25$ & $87.1[83.7-89.9]$ & $31.3[27.2-35.4]$ & $48.3[43.9-52.7]$ & $5.9[3.8-8.0]$ & $1.0[0.1-1.9]$ \\
\hline $26-35$ & $88.4[85.8-90.7]$ & $36.7[33.0-40.4]$ & $40.6[36.8-44.4]$ & $7.8[5.8-9.8]$ & $2.7[1.5-3.9]$ \\
\hline $36-45$ & $81.7[77.7-85.2]$ & $36.1[31.6-40.6]$ & 36.8 [32.3-41.3] & $7.8[5.3-10.3]$ & $1.6[0.4-2.8]$ \\
\hline $46-55$ & $86.6[82.1-90.1]$ & $33.9[28.5-39.3]$ & $41.9[36.3-47.5]$ & $9.7[6.3-13.1]$ & $2.6[0.8-4.4]$ \\
\hline $56-65$ & $78.4[72.3-83.6]$ & $34.4[28.1-40.7]$ & $33.9[27.6-40.2]$ & $8.3[4.6-12.0]$ & $3.7[1.2-6.2]$ \\
\hline \multicolumn{6}{|c|}{ Household consumption (USD/year) } \\
\hline $950-1,200$ & $85.2[82.6-87.6]$ & $34.4[31.1-37.7]$ & $41.4[38.0-44.8]$ & $7.3[5.5-9.1]$ & $2.2[1.2-3.2]$ \\
\hline$<950$ & 83.5 [80.7-85.9] & 35.2 [31.9-38.5] & $39.2[35.9-42.2]$ & $7.2[5.4-9.0]$ & $1.5[0.7-2.3]$ \\
\hline$>1,200$ & 89.2 [85.9-91.7] & 34.3 [30.0-38.6] & $43.9[39.4-48.4]$ & $9.1[6.5-11.7]$ & $3.4[1.8-5.0]$ \\
\hline \multicolumn{6}{|l|}{ Habitation } \\
\hline Rural & $83.4[81.3-85.4]$ & $35.2[32.6-37.7]$ & $38.0[35.4-40.6]$ & $8.1[6.6-9.6]$ & $2.0[1.3-2.7]$ \\
\hline Urban & 88.9 [86.4-90.9] & $33.8[30.5-37.1]$ & $46.5[43.0-50.0]$ & $7.0[5.1-8.8]$ & $2.6[1.5-3.7]$ \\
\hline \multicolumn{6}{|c|}{ Household altitude } \\
\hline$<1,000 \mathrm{~m}$ & 84.5 [82.3-86.7] & $28.7[25.9-31.5]$ & $46.4[43.3-49.5]$ & $8.2[6.5-9.9]$ & $1.8[1.0-2.6]$ \\
\hline$\geq 1,000 \mathrm{~m}$ & 86.3 [84.3-88.3] & $40.1[37.2-43.0]$ & 36.3 [33.5-39.1] & 9.7 [7.9-11.4] & $2.5[1.6-3.4]$ \\
\hline
\end{tabular}

Table 3 Bivariate logistic regression analyses of associations of each headache type with gender, age, household consumption, habitation and altitude

\begin{tabular}{|c|c|c|c|c|c|c|}
\hline & \multicolumn{2}{|l|}{ Migraine } & \multicolumn{2}{|c|}{ Tension-type headache } & \multicolumn{2}{|c|}{ Probable medication-overuse headache } \\
\hline & $\mathrm{OR}[95 \% \mathrm{Cl}]$ & $p$ & OR $[95 \% \mathrm{Cl}]$ & $p$ & OR $[95 \% \mathrm{Cl}]$ & $p$ \\
\hline \multicolumn{7}{|l|}{ Gender } \\
\hline Male & Reference & - & Reference & - & Reference & - \\
\hline Female & $1.6[1.3-1.9]$ & $<0.001$ & $0.7[0.6-0.9]$ & 0.002 & $2.2[1.1-4.4]$ & 0.020 \\
\hline \multicolumn{7}{|l|}{ Age (years) } \\
\hline $18-25$ & Reference & - & Reference & - & Reference & - \\
\hline $26-35$ & $1.3[0.99-1.6]$ & 0.057 & $0.7[0.6-0.9]$ & 0.010 & $2.7[1.01-7.4]$ & 0.049 \\
\hline $36-45$ & $1.2[0.9-1.6]$ & 0.12 & $0.6[0.5-0.8]$ & $<0.001$ & $1.6[0.5-4.9]$ & 0.44 \\
\hline $46-55$ & $1.1[0.8-1.5]$ & 0.45 & $0.8[0.6-1.1]$ & 0.085 & $2.7[0.9-8.2]$ & 0.088 \\
\hline $56-65$ & $1.1[0.8-1.6]$ & 0.41 & $0.6[0.4-0.8]$ & $<0.001$ & $3.7[1.2-11.4]$ & 0.023 \\
\hline \multicolumn{7}{|c|}{ Household consumption (USD/year) } \\
\hline $950-1,200$ & Reference & - & Reference & - & Reference & - \\
\hline$<950$ & $1.1[0.8-1.3]$ & 0.74 & $0.9[0.7-1.1]$ & 0.35 & $0.6[0.3-1.4]$ & 0.25 \\
\hline$>1,200$ & $0.99[0.8-1.3]$ & 0.99 & $1.1[0.9-1.4]$ & 0.40 & $1.5[0.8-3.0]$ & 0.22 \\
\hline \multicolumn{7}{|l|}{ Habitation } \\
\hline Rural & Reference & - & Reference & - & Reference & - \\
\hline Urban & $0.9[0.8-1.1]$ & 0.53 & $1.4[1.2-1.7]$ & $<0.001$ & $1.3[0.7-2.4]$ & 0.34 \\
\hline \multicolumn{7}{|c|}{ Household altitude } \\
\hline$<1,000 \mathrm{~m}$ & Reference & - & Reference & - & Reference & - \\
\hline$\geq 1,000 \mathrm{~m}$ & $1.7[1.4-2.0]$ & $<0.001$ & $0.7[0.6-0.8]$ & $<0.001$ & $1.4[0.8-2.6]$ & 0.25 \\
\hline
\end{tabular}


Table 4 Multivariate logistic regression analyses of associations of each headache type ${ }^{a}$

\begin{tabular}{|c|c|c|c|c|c|c|}
\hline & \multicolumn{2}{|l|}{ Migraine } & \multicolumn{2}{|c|}{ Tension-type headache } & \multicolumn{2}{|c|}{ Probable medication-overuse headache } \\
\hline & AOR $[95 \% \mathrm{Cl}]$ & $p$ & $\mathrm{AOR}[95 \% \mathrm{Cl}]$ & $p$ & $\mathrm{AOR}[95 \% \mathrm{Cl}]$ & $p$ \\
\hline \multicolumn{7}{|l|}{ Gender } \\
\hline Male & Reference & - & Reference & - & Reference & - \\
\hline Female & $1.5[1.2-1.8]$ & $<0.001$ & $0.7[0.6-0.9]$ & 0.003 & $2.6[1.2-5.3]$ & 0.010 \\
\hline \multicolumn{7}{|l|}{ Age (years) } \\
\hline $18-25$ & Reference & - & Reference & - & Reference & - \\
\hline $26-35$ & $1.3[1.0-1.6]$ & 0.060 & $0.8[0.6-0.9]$ & 0.023 & $2.7[0.9-7.5]$ & 0.054 \\
\hline $36-45$ & $1.2[0.9-1.6]$ & 0.19 & $0.7[0.5-0.8]$ & 0.004 & $1.5[0.5-4.8]$ & 0.52 \\
\hline $46-55$ & $1.1[0.8-1.4]$ & 0.75 & $0.9[0.7-1.2]$ & 0.44 & $2.1[0.7-6.8]$ & 0.21 \\
\hline $56-65$ & $1.1[0.8-1.6]$ & 0.57 & $0.6[0.4-0.9]$ & 0.008 & $2.6[0.8-8.8]$ & 0.11 \\
\hline \multicolumn{7}{|c|}{ Household consumption (USD/year) } \\
\hline $950-1,200$ & Reference & - & Reference & - & Reference & - \\
\hline$<950$ & $1.0[0.8-1.2]$ & 0.94 & $1.0[0.8-1.2]$ & 0.76 & $0.6[0.3-1.2]$ & 0.16 \\
\hline$>1,200$ & $1.1[0.8-1.4]$ & 0.66 & $1.1[0.8-1.2]$ & 0.58 & $1.3[0.7-2.4]$ & 0.44 \\
\hline \multicolumn{7}{|l|}{ Habitation } \\
\hline Rural & Reference & - & Reference & - & Reference & - \\
\hline Urban & $1.0[0.8-1.2]$ & 0.81 & $1.3[1.1-1.6]$ & 0.003 & $1.3[0.7-2.4]$ & 0.44 \\
\hline \multicolumn{7}{|c|}{ Household altitude } \\
\hline$<1,000 \mathrm{~m}$ & Reference & - & Reference & - & Reference & - \\
\hline$\geq 1,000 \mathrm{~m}$ & $1.6[1.3-2.0]$ & $<0.001$ & $0.7[0.6-0.8]$ & $<0.001$ & $1.4[0.8-2.6]$ & 0.29 \\
\hline
\end{tabular}

adjusted for gender, age, household consumption, household altitude, systolic and diastolic blood pressure, EPQRS-N and HADS scores; AOR adjusted odds ratio, $\mathrm{Cl}$ confidence interval

1.2-1.7]; $p=0.001 ;$ AOR $=1.3$ [95 \% CI: 1.1-1.6]; $p=$ 0.003 ) (Tables 3 and 4). Migraine was strongly associated with living at an altitude of $\geq 1,000 \mathrm{~m}$ in both bivariate $(\mathrm{OR}=1.7 \quad[95 \quad \% \quad \mathrm{CI}: \quad 1.4-2.0] ; \quad p<0.001)$ (Table 3) and multivariate analyses $(\mathrm{AOR}=1.6[95 \%$ CI: $1.3-2.0] ; p<0.001$ ) (Table 4 ). In view of this finding, we estimated the prevalence of migraine by altitude category, standardising for age and gender against census data for the Nepali population [20] (Table 5). This analysis revealed that the age- and gender standardised prevalence of migraine was $27.9 \%$ in the low-lying Terai $(<500 \mathrm{~m}$; there were no participants in our sample living between 500 and $999 \mathrm{~m})$. Prevalence increased in an almost linear relationship with altitude up to $2,000 \mathrm{~m}$. Thereafter it levelled, and indeed declined. This relationship was evident in both genders.

A negative association between TTH and altitude was indicated by both bivariate $(\mathrm{OR}=0.7$ [95 \% CI: 0.6-0.8]; $p<0.001)$ and multivariate analyses $(\mathrm{AOR}=0.7$ [95\% CI: 0.6-0.8]; p < 0.001) (Tables 3 and 4).

\section{Discussion}

We found a gender- and age adjusted 1-year prevalence of all headache of $84.9 \%$, of migraine $34.1 \%$, of TTH $41.5 \%$, of all headache on $\geq 15$ days/month $7.4 \%$, and of
pMOH $2.1 \%$. We showed that living at an altitude of $\geq 1,000 \mathrm{~m}$ was highly associated with migraine, and urban dwelling was less strongly associated with TTH.

Before discussing the individual headache types, we note that our study used tried and tested methods [21]. We randomly selected from the whole of Nepal, while the very high participation rate ( $>99 \%)$ achieved through careful methodology $[10,15]$ effectively excluded participation bias. Face-to-face interviews were conducted carefully to ensure there were no missing data. These were considerable strengths of the study. There were, however, some important limitations, which we will draw attention to in the context of their relevance.

\section{Prevalence of migraine}

Our most obvious finding was that the prevalence of migraine in Nepal is very much higher than the mean global estimate of $14.7 \%$ [22]. Our first comment on this is to note that more recent studies, in all regions except the Far East, have generally yielded higher values than $14.7 \%$. The mean global estimate is based on a large number of heterogeneous studies, performed with varying methods during a period of $>30$ years. Many reports are silent on the crucial issue of how they applied diagnostic criteria with 
Table 5 Observed and age- and gender-standardised prevalence of migraine by altitude and gender

\begin{tabular}{|c|c|c|c|c|c|c|c|}
\hline \multirow{2}{*}{$\begin{array}{l}\text { Physiographic } \\
\text { division and altitude }\end{array}$} & \multicolumn{4}{|c|}{ Observed prevalence in sample } & \multicolumn{3}{|c|}{ Standardised prevalence* [20] } \\
\hline & N & All $n(\%)$ & Male $n(\%)$ & Female $n(\%)$ & All \% & Male \% & Female $\%$ \\
\hline Terai $<500 \mathrm{~m}$ & 1,000 & $287(28.7)$ & $86(22.0)$ & $201(33.0)$ & 27.9 & 22.1 & 32.9 \\
\hline \multicolumn{8}{|l|}{ Hill } \\
\hline $1,000-1,499 \mathrm{~m}$ & 470 & $176(37.7)$ & $57(31.1)$ & $119(41.5)$ & 36.5 & 31.6 & 40.7 \\
\hline $1,500-1,999 \mathrm{~m}$ & 160 & $68(42.5)$ & $31(39.7)$ & $37(45.1)$ & 44.4 & 41.3 & 47.0 \\
\hline \multicolumn{8}{|l|}{ Mountain } \\
\hline $2,000-2,499 \mathrm{~m}$ & 254 & $116(45.7)$ & $46(41.1)$ & $70(49.3)$ & 45.5 & 40.8 & 49.6 \\
\hline$\geq 2,500 \mathrm{~m}$ & 216 & $81(37.5)$ & 29 (29.9) & $52(43.7)$ & 37.9 & 31.4 & 43.6 \\
\hline
\end{tabular}

${ }^{*}$ Age- and gender-standardised against census data for the Nepali population [20]

respect to definite and probable migraine; while some explicitly excluded the latter, it is highly probable that more did so without making this evident, because only recently has there been clear guidance and explanation of why this is misleading [21]. The consequences, in our view, are that many of these studies significantly underestimate migraine prevalence and, therefore, so does the global mean. Nonetheless, our finding of $34.1 \%$ in Nepal is considerably higher even than the $25.2 \%$ reported from neighbouring India [23] (although this was from the single State of Karnataka in the south). We used the same methodology and diagnostic questionnaire as Karnataka [24]; indeed, LTB has supported studies using similar methods and the same questionnaire in many other countries, cultures and languages [13]: in Russia [25], China [26], Zambia [27], Ethiopia (unpublished), Pakistan (unpublished), Saudi Arabia (unpublished), Morocco (unpublished). None has discovered such a high prevalence of migraine; in fact, India (Karnataka) was the highest [23].

Nepal is a poor country, but we discovered no association with economic wellbeing that might offer an explanation (neither was there one in India [23]). The clearly relevant factor is altitude: over half our sample were resident at or above $1,000 \mathrm{~m}$, and nearly one quarter above 2,000 m. Altitude, we discovered, is an environmental factor so strongly associated with migraine that living above $1,000 \mathrm{~m}$ led to $60 \%$ increased odds of having the disorder. In practice this meant that, among the adult population living above this altitude, an additional $11.4 \%$ had migraine (Table 2). Since more than half the population were in fact living above $1,000 \mathrm{~m}$, this explained a very large part of the overall excess prevalence. For the remainder, we should look to the distinctive age and gender distributions of Nepal: more precisely, to the high proportions of young adults (median age is 22 years [9]), and females among these adults, the two strongest determinants of migraine prevalence [1]. Furthermore, large numbers of healthy people have consistently been going abroad for work, around half of them in the age group 15-35 years, and mostly males [9], leaving behind a skewed adult distribution perhaps more at risk of migraine. It is entirely possible to see this as the explanation of the difference between $27.9 \%$, the standardised prevalence in the Terai of Nepal, and $25.2 \%$, the standardised prevalence in Karnataka [23] (which is at a mean altitude of about $900 \mathrm{~m} \mathrm{[28]).}$

We should acknowledge two diagnostic issues. First, it was a limitation of the study that we were not able to perform a prior validation of the headache diagnostic questionnaire in its Nepali translation. Because there are no headache specialists in the country, we had no means of applying a "gold standard" for this purpose [21]. We had to rely on the fact that the questionnaire had been validated in many other languages and countries [13], including India with not too dissimilar cultural settings [24].

Second, having said this, we encountered a problem that was not met in India: such a large proportion (75.8\%) of respondents with any type of headache reported photophobia that this symptom had virtually no discriminative value as a diagnostic criterion, and we could not use it within the framework of ICHD [18]. Photophobia is a technical concept, not easy to convey to lay participants (even by trained interviewers) [21]. Because of this we had taken great care in translation to convey not merely an aversion to bright light but the idea of a wish to withdraw into darkness from ordinary light. Our eventual solution was to disregard photophobia altogether, and in our view this was necessary: the prevalence estimate for migraine would otherwise have been much higher. What this suggests is that ICHD criteria - or at least this particular one - may not be universally applicable, and not for linguistic reasons.

\section{Other headache types}

The estimated prevalence of TTH in Nepal at $41.1 \%$ is in line with the estimated global average [2], although considerably lower than some national estimates [29, 30]. 
Two factors are relevant here. One is whether or not survey participants report infrequent $\mathrm{TTH}$, which can have a marked effect on the prevalence estimate and is likely to be determined in part culturally and in part by the insistence (and purpose) of the interviewer [23, 24]. In our case, not only did we not consider infrequent TTH to be of public-health significance, but also we focused only on the most bothersome headache in participants identifying more than one type. Those with both migraine and TTH would tend to regard the former as the more bothersome [31], leading to a partial neglect of TTH. Our prevalence estimate for TTH was therefore somewhat conservative.

We suspect this was a factor in the observed negative association between TTH prevalence and altitude: as migraine prevalence increased with altitude, reporting of TTH became less likely. In other words, it was artefactual. We do not know this, but on the other hand can offer no explanation for a true negative association.

TTH was significantly more prevalent in urban areas, an association not observed for migraine or $\mathrm{pMOH}$. Possible explanations for the higher prevalence of TTH may be the noisy and stressful environments of the cities. Also, physical inactivity is a risk factor specifically for non-migraine headache [32], and people in rural areas in Nepal are more physically active working in the fields. These are speculative proposals.

According to our estimate, the prevalence of headache on $\geq 15$ days/month in Nepal is more than twice the global average [2] and much higher than in neighbouring China [26] and India [23]. Several factors such as low socio-economic status, poor access to health services with a paucity of health-care providers and lack of standard protocols for diagnosis and treatment of headache disorders, all against a background of high levels of episodic headache, offer what may be sufficient explanation.

We do not know what the cases with headache on $\geq 15$ days/month were diagnostically, other than the proportion diagnosed as $\mathrm{pMOH}$. We estimated the prevalence of $\mathrm{pMOH}$ in Nepal at $2.1 \%$, which is towards the upper end of the range for most countries studied $(0.5-2.6 \%)$ [33], and much higher than the $0.6 \%$ and $1.2 \%$ in neighbouring China [26] and India [23]. Surprisingly, the proportion of $\mathrm{pMOH}$ among those with headache on $\geq 15$ days/month was only $29 \%$ (46/161); in other countries where studies have used similar methodology, this proportion is closer to two thirds $[23,25,26]$. This may be due to poverty: most $\mathrm{pMOH}$ was associated with overuse of simple analgesics sold over-the-counter (OTC), but many people in Nepal cannot afford these. They have recourse to alternative treatments, which may include plant-based remedies that are used regularly across the country, especially in the rural areas. Many of these have phytochemical or pharmacological properties [34], and may be able to cause transformation of episodic headache to $\mathrm{pMOH}$. We endeavoured to include these in our enquiry, and identified six cases, but herb-based remedies may not be perceived by lay people as therapy for headache and it is likely that they were underreported.

\section{Implications for Nepal}

Headache disorders are very common in Nepal. Only the prevalence of TTH is in line with the global average. That of headache on $\geq 15$ days/month is double, while the prevalence of $\mathrm{pMOH}$ is towards the upper end of the range for most countries studied. The prevalence of migraine, however, appears uniquely high, explained, we believe, by some of the distinct characteristics of this country. One is the combination of mountainous and hilly terrain over much of the country, important in the light of the previously unreported but strong association between migraine prevalence and altitude. Another is the demographic make-up.

The new evidence from this study will inform national health policy, and provide a basis for health-care needs assessment. Meanwhile, research of a different type is needed to find explanations for the association between migraine and altitude, since these may be relevant to health-care interventions. Continuous long-duration exposure to high altitude compromises oxygen uptake and results in haemodynamic changes, with elevated haemoglobin levels, increased blood viscosity and reduced oxygen delivery to brain tissues [35, 36]. Migraine has been linked with the consequences of such changes [37-40], but their relevance at lower altitudes, between 500 and $2,000 \mathrm{~m}$, needs to be investigated.

\section{Conclusion}

Headache disorders are very common in Nepal. Migraine is unusually so, and strongly associated with altitude of dwelling, which in this mountainous country largely explains the high national prevalence. Headache occurring on $\geq 15$ days/month is also very common in comparison with the world average. This new evidence will inform national health policy and provide a basis for health-care needs assessment. However, research is needed to explain the association of migraine with altitude, since it may be relevant to health-care interventions.

\section{Abbreviations}

AOR: Adjusted odds ratio; BMI: Body-mass index; BP: Blood pressure; $\mathrm{Cl}$ : Confidence interval; EPQRS-N: Eysenck Personality Questionnaire Short Form Revised version- Neuroticism; GBD: Global burden of disease; HADS: Hospital Anxiety and Depression Scale; HARDSHIP: Headache-Attributed Restriction, Disability, Social Handicap and Impaired Participation; ICHD: International classification of headache disorders; IRC-KUSMS: The Institutional Review Committee of Kathmandu University School of Medical 
Sciences, Dhulikhel Hospital; LTB: Lifting The Burden; MOH: Medication-overuse headache; NPR: Nepalese rupee; OR: Odds ratio; OTC: Over-the-counter; pMOH: Probable MOH; SEAR: South-East Asia Region; SPSS: Statistical package for social science; TTH: Tension-type headache; USD: United States dollar; YLD: Year of life lost to disability.

\section{Competing interests}

TJS is a Director and Trustee of Lifting The Burden.

\section{Authors' contributions}

All authors were involved in conception and design of the project. KM, AR, TJS and ML took part in acquisition, analysis and interpretation of the data. KM drafted the manuscript. All authors reviewed it critically for intellectual content and approved the final version of the manuscript.

\section{Acknowledgements}

This project was funded by grants from Samarbeidsorganet, the Liaison Committee between the Central Norway Regional Health Authority and the Norwegian University of Science and Technology (NTNU). It was conducted within the Global Campaign against Headache, led by the nongovernmental organization Lifting The Burden in official relations with the World Health Organization, and with the support of Dhulikhel Hospital, Kathmandu University Hospital, Dhulikhel, Kavre, Nepal.

\section{Author details}

'Department of Neuroscience, Norwegian University of Science and Technology, St Olavs University Hospital, Trondheim, Norway. ${ }^{2}$ Dhulikhel Hospital, Kathmandu University Hospital, Kavre, Dhulikhel, Nepal. ${ }^{3}$ Division of Brain Sciences, Imperial College London, London, UK. ${ }^{4}$ Pain Unit, St Olavs University Hospital, Trondheim, Norway. ${ }^{5}$ Norwegian Advisory Unit on Headaches, St Olavs University Hospital, Trondheim, Norway.

\section{Received: 21 September 2015 Accepted: 6 November 2015 Published online: 10 November 2015}

\section{References}

1. Jensen R, Stovner $\sqcup$ (2008) Epidemiology and comorbidity of headache. Lancet Neurol 7(4):354-361. doi:10.1016/s1474-4422(08)70062-0

2. Stovner L, Hagen $K$, Jensen R, Katsarava Z, Lipton R, Scher A, et al (2007) The global burden of headache: a documentation of headache prevalence and disability worldwide. Cephalalgia 27(3):193-210. doi:10.1111/j.1468-2982.2007.01288.x

3. Linde M, Gustavsson A, Stovner LJ, Steiner TJ, Barre J, Katsarava Z, et al (2012) The cost of headache disorders in Europe: the Eurolight project. Eur J Neurol 19(5):703-711. doi:10.1111/j.1468-1331.2011.03612.x

4. Vos T, Barber RM, Bell B, Bertozzi-Villa A, Biryukov S, Bolliger l, et al (2015) Global, regional, and national incidence, prevalence, and years lived with disability for 301 acute and chronic diseases and injuries in 188 countries, 1990-2013: a systematic analysis for the Global Burden of Disease Study 2013. Lancet 386:743-800. doi:10.1016/s0140-6736(15)60692-4

5. Steiner TJ, Birbeck GL, Jensen RH, Katsarava Z, Stovner LJ, Martelletti $P$ (2015) Headache disorders are third cause of disability worldwide. $J$ Headache Pain 16:58. doi:10.1186/s10194-015-0544-2

6. World Health Organization and Lifting The Burden (2011) Atlas of headache disorders and resources in the world 2011. WHO, Geneva

7. United States Central Intelligence Agency, Office of Public Affairs. The World Factbook. https://www.cia.gov/library/publications/the-world-factbook/geos/ np.html. Accessed 19 Sep 2015.

8. Government of Nepal, National Planning Commission Secretariate (2014) Population Monograph of Nepal, Vol I. Central Bureau of Statistics Kathmandu, Nepal

9. Government of Nepal, National Planning Commission Secretariate (2014) Population monograph of Nepal, vol II. Central Bureau of Statistics, Kathmandu, Nepal

10. Manandhar K, Risal A, Steiner TJ, Holen A, Koju R, Linde M (2014) Estimating the prevalence and burden of major disorders of the brain in Nepal: methodology of a nationwide population-based study. J Headache Pain 15:52. doi:10.1186/1129-2377-15-52

11. Bhattarai B, Pokhrel PK, Tripathi M, Rahman TR, Baral DD, Pande R, et al (2007) Chronic pain and cost: an epidemiological study in the communities of Sunsari district of Nepal. Nepal Med Coll J 9(1):6-11
12. Wright C, Nepal MK, Bruce-Jones WD (1989) Mental health patients in primary health care services in Nepal. Asia Pac J Public Health 3(3):224-230

13. Steiner TJ, Gururaj G, Andree C, Katsarava Z, Ayzenberg I, Yu SY, et al (2014) Diagnosis, prevalence estimation and burden measurement in population surveys of headache: presenting the HARDSHIP questionnaire. J Headache Pain 15:3. doi:10.1186/1129-2377-15-3

14. Peters M, Bertolote JM, Houchin C, Kandoura T, Steiner TJ (2007) Translation protocol for hybrid documents. J Headache Pain 8(Suppl 1):S40-S47

15. Risal A, Manandhar K, Steiner TJ, Holen A, Koju R, Linde M (2014) Estimating prevalence and burden of major disorders of the brain in Nepal: cultural, geographic, logistic and philosophical issues of methodology. J Headache Pain 15:3. doi:10.1186/1129-2377-15-51

16. Risal A, Manandhar K, Linde M, Koju R, Steiner TJ, Holen A. Reliability and validity of a Nepali-language version of the Hospital Anxiety and Depression Scale (HADS). Kathmandu Univ Med J (In press).

17. Manandhar K, Risal A, Linde M, Koju R, Steiner TJ, Holen A. Measuring neuroticism in Nepali: Reliability and validity of the Neuroticism Subscale of the Eysenck Personality Questionnaire. Kathmandu Univ Med J (In press).

18. International Headache Society Classification Commitee (2013) The International Classification of Headache Disorders, 3rd edition (beta version). Cephalalgia 33(9):629-808. doi:10.1177/0333102413485658

19. Linde M, Dahlof C (2004) Attitudes and burden of disease among selfconsidered migraineurs-a nation-wide population-based survey in Sweden. Cephalalgia 24(6):455-465. doi:10.1111/j.1468-2982.2004.00703.x

20. Government of Nepal, National Planning Commission Secretariate (2012) Population and Housing Census 2011. Central Bureau of Statistics, Kathmandu, Nepal

21. Stovner LJ, Al Jumah M, Birbeck GL, Gururaj G, Jensen R, Katsarava Z, et al (2014) The methodology of population surveys of headache prevalence, burden and cost: Principles and recommendations from the Global Campaign against Headache. J Headache Pain 15:5 doi:10.1186/1129-2377-15-5

22. Vos T, Flaxman AD, Naghavi M, Lozano R, Michaud C, Ezzati M, et al (2012) Years lived with disability (YLDs) for 1160 sequelae of 289 diseases and injuries 1990-2010: a systematic analysis for the Global Burden of Disease Study 2010. Lancet 380:2163-2196. doi:10.1016/s0140-6736(12)61729-2

23. Kulkarni GB, Rao GN, Gururaj G, Stovner LJ, Steiner TJ (2015) Headache disorders and public ill-health in India: prevalence estimates in Karnataka State. J Headache Pain 16:67. doi:10.1186/s10194-015-0549-x

24. Rao GN, Kulkarni GB, Gururaj G, Rajesh K, Subbakrishna DK, Steiner TJ, et al (2012) The burden of headache disorders in India: methodology and questionnaire validation for a community-based survey in Karnataka State. J Headache Pain 13(7):543-550. doi:10.1007/s10194-012-0474-1

25. Ayzenberg I, Katsarava Z, Sborowski A, Chernysh M, Osipova V, Tabeeva G, et al (2012) The prevalence of primary headache disorders in Russia: a countrywide survey. Cephalalgia 32(5):373-381. doi:10.1177/0333102412438977

26. Yu S, Liu R, Zhao G, Yang X, Qiao X, Feng J, et al (2012) The prevalence and burden of primary headaches in China: a population-based door-to-door survey. Headache 52(4):582-591

27. Mbewe E, Zairemthiama P, Yeh HH, Paul R, Birbeck GL, Steiner TJ (2015) The epidemiology of primary headache disorders in Zambia: a population-based door-to-door survey. J Headache Pain 16:30. doi:10.1186/s10194-015-0515-7

28. Swaminathan JM (2009) Indian economic superpower: fiction or future? World Scientific: p 20. ISBN 9789812814661.

29. Rasmussen BK, Jensen R, Schroll M, Olesen J (1991) Epidemiology of headache in a general population-a prevalence study. J Clin Epidemiol 44(11):1147-1157

30. Lyngberg AC, Rasmussen BK, Jorgensen T, Jensen R (2005) Has the prevalence of migraine and tension-type headache changed over a 12-year period? A Danish population survey. Eur J Epidemiol 20(3):243-249

31. Krogh $A B$, Larsson $B$, Linde $M$ (2015) Prevalence and disability of headache among Norwegian adolescents: A cross-sectional school-based study. Cephalalgia. doi:10.1177/0333102415573512

32. Varkey E, Hagen K, Zwart JA, Linde M (2008) Physical activity and headache: results from the Nord-Trondelag Health Study (HUNT). Cephalalgia 28(12):1292-1297. doi:10.1111/j.1468-2982.2008.01678.x

33. Westergaard ML, Hansen EH, Glumer C, Olesen J, Jensen RH (2014) Definitions of medication-overuse headache in population-based studies 
and their implications on prevalence estimates: a systematic review. Cephalalgia 34(6):409-425. doi:10.1177/0333102413512033

34. Uprety Y, Asselin H, Boon EK, Yadav S, Shrestha KK (2010) Indigenous use and bio-efficacy of medicinal plants in the Rasuwa District, Central Nepal. J Ethnobiol Ethnomed 6:3. doi:10.1186/1746-4269-6-3

35. Hackett PH, Roach RC (2001) High-altitude illness. N Engl J Med 345(2):107-114. doi:10.1056/nejm200107123450206

36. Queiroz LP, Rapoport AM (2007) High-altitude headache. Curr Pain Headache Rep 11(4):293-296

37. Arregui A, Leon-Velarde F, Cabrera J, Paredes S, Vizcarra D, Umeres H (1994) Migraine, polycythemia and chronic mountain sickness. Cephalalgia 14(5):339-341

38. Lippi G, Cervellin G, Mattiuzzi C (2014) Migraine and erythrocyte biology: a review. Int J Lab Hematol 36(6):591-597. doi:10.1111/ijlh.12199

39. Hagen K, Wisloff U, Ellingsen O, Stovner L, Linde M (2015) Headache and peak oxygen uptake: The HUNT3 study. Cephalalgia 35 (on-line publication) doi:10.1177/0333102415597528.

40. Aamodt AH, Borch-lohnsen B, Hagen K, Stovner LJ, Asberg A, Zwart JA (2004) Headache prevalence related to haemoglobin and ferritin. The HUNT Study. Cephalalgia 24(9):758-762. doi:10.1111/j.1468-2982.2004.00756.x

Submit your manuscript to a SpringerOpen ${ }^{\circ}$ journal and benefit from:

- Convenient online submission

- Rigorous peer review

- Immediate publication on acceptance

- Open access: articles freely available online

- High visibility within the field

- Retaining the copyright to your article

Submit your next manuscript at $>$ springeropen.com 